\section{Human auditory evoked response intensity functions from monopolar and bipolar scalp recordings*}

\author{
R. L. KLINGAMAN† and A. M. ANCH†† \\ Saint Louis University, St. Louis, Mo. 63103
}

Average evoked brain responses (AER) were computed from two stimultaneous recordings during binaural tone stimulation for three Ss. Linear regressions were then fitted to the data, and correlation coefficients also were computed. The results showed that, for the monopolar $\left(C_{3}-A_{2}\right)$ recording, a linear relationship between sound level and size of AER was found when the Ss' data were pooled, with both components showing this relationship. The bipolar recording $(\mathrm{Cz}-\mathrm{Oz})$ showed a linear relationship for one component but not for the other. The slope of the AER component showing an intensity function was the same for both recording sites.

The question of whether a monopolar or a bipolar recording is "better" has been extensively debated in the research literature, but little empirical evidence has been offered. Very few studies have compared evoked brain responses (EBR) recorded simultaneously from a monopolar configuration with a similarly recorded bipolar configuration. Goff et al (1969) reports that, for the visual EBR, the bipolar recording tends to give waveforms that are more variable and to introduce distortions not present in the monopolar recording. Vaughan (1966) also reports similar results for the visual EBR. On the other hand, Perry \& Childers (1969) feel that there are few differences between the two recording methods. These studies have limited themselves to presenting either one or two intensity levels of the sensory stimulus. We felt that by using a wider range of stimulus intensities, a more sensitive and accurate picture of the effect of a recording parameter manipulation could be assessed by comparing the obtained intensity functions.

A recent study, done in this lab (Tepas et al, 1972) compared intensity functions derived from four bipolar electrode sites. These sites, as per the international electrode system (Jasper, 1958) were $\mathrm{Fz} \cdot \mathrm{Pz}, \mathrm{Cz} \cdot \mathrm{Fz}, \mathrm{Cz} \cdot \mathrm{Oz}$, and $\mathrm{Pz}-\mathrm{Oz}$. An auditory stimulus whose intensity was varied in 2-dB steps over a $20-\mathrm{dB}$ range, with the highest

\footnotetext{
*This study was partially supported by Research Grant MH 14020-03 from the National Institute of Mental Health to Donald I. Tepas.

tRequests for reprints should be sent to R. L. Klingaman, Department of Psychology. St. Louis University, St. Louis, Mo. 63103 .

††Present address: Department of Psychiatry and Behavior, University of Oklahoma Medical Center, Oklahoma City, Okla. 73104 .
} frequently in sleep studies vertex.
(Rechtschaffen \& Kales, 1968) to one $(\mathrm{Cz} \cdot \mathrm{Oz})$ used in many awake studies; and (3) to ascertain the "goodness of fit" of the linear model over a wider range of stimulus intensities. SUBJECTS

Ss were three male adults (average age $=21.6$ years) with normal hearing, as tested by an AMBCO Model A-17 audiometer. The Ss were concurrently participating in a second study dealing with auditory EBRs collected during all-night sleep. The Ss were tested in the present experiment shortly before going to sleep for the night. APPARATUS

Auditory stimuli were generated by the modular system described by Tepas et al (1972) and were delivered to the Ss through Gentex earphones (GH-138). A tone burst of $1-\mathrm{kHz}$ frequency was presented binaurally at a $1 / \mathrm{sec}$ rate. The tone's duration was $30 \mathrm{msec}$, with a 10 -msec rise-fall time. Calibration of the Gentex earphones was done psychophysically, using as a standard a TDH 39 (Telephonics) earphone that had been calibrated by using a Bruel and Kjaer Model 158 audiometer calibrator with a NBS9A 6-c c acoustic coupler. The psychophysical calibration was done by equating the loudness of each of the two Gentex earphones, using as a standard the TDH 39 earphone. Both ascending and descending series in $1-d B$ steps of attenuation were used in matching for loudness. A direct calibration, using the audiometer calibrator, was not practical since the Gentex earphones have nonstandard

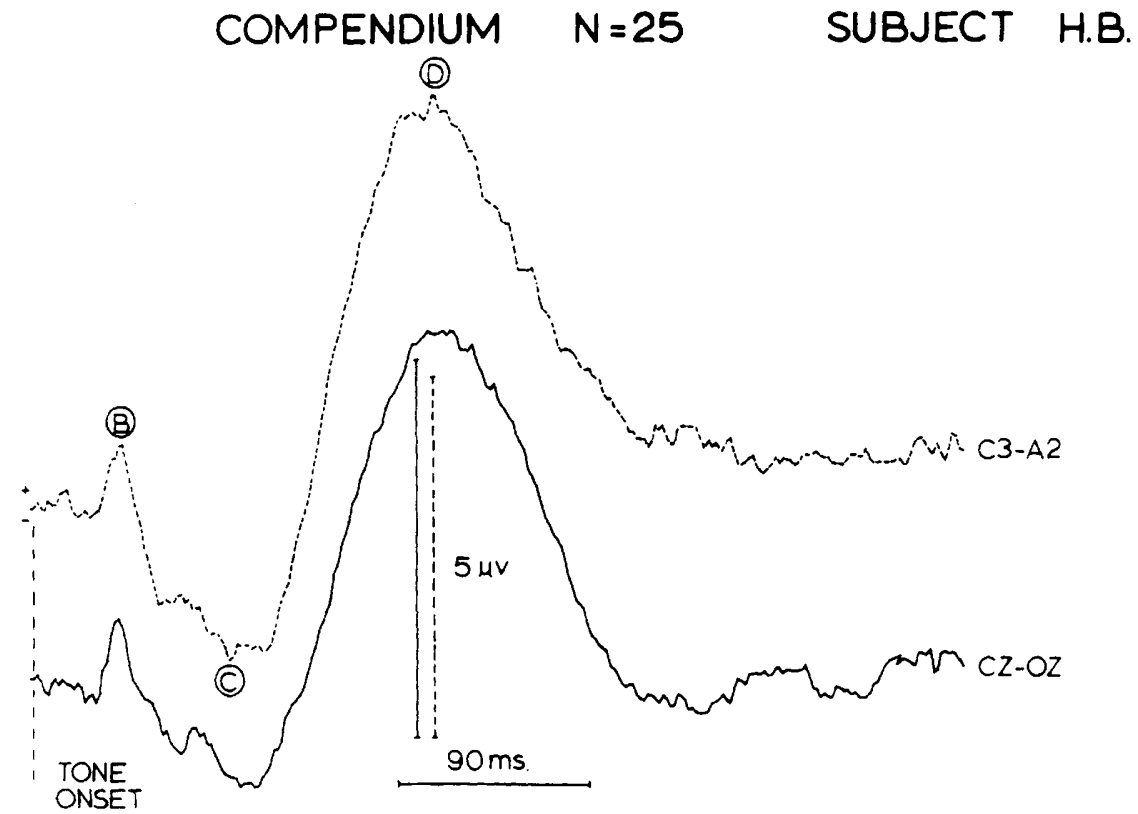

Fig. 1. Two compendiums for S H.B. Each compendium represents the sum of 25 trials. A positive voltage causes an upward deflection with respect to the 


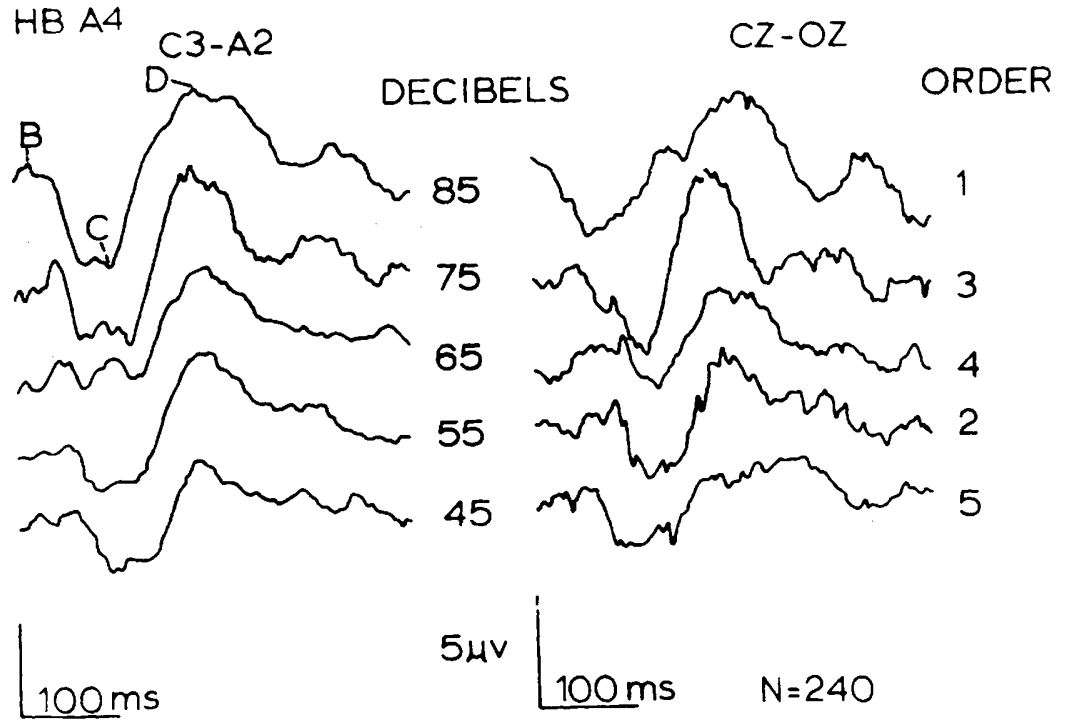

Fig. 2. Analog data plots of the individual EBRs for a single session. The $S$ is H.B. The order in which the stimuli were presented is noted at the right.

ear cushions and headset. The headset assembly was fabricated out of a stretch nylon material (Lycra) and had an adjustable chin strap. The assembly resembled a helmet and was designed and built by the Gentex Co. ${ }^{2}$ The same system was worn during the presleep awake study and the subsequent sleep study.

Grass E5S electrodes were used to record the electroencephalogram (EEG). They were fastened to the scalp with collodion, at Positions $\mathrm{Cz}$, $\mathrm{C}_{3}$, and $\mathrm{Oz}$. Beckman 11-mm biopotential electrodes, attached to left and right mastoid, served as indifferent (left mastoid) and ground (right mastoid) for the recording. A Grass Model 7 polygraph, equipped with P5A preamplifiers, was used to monitor and amplify the EEG continuously. Amplified potentials from the driver amplifiers were then fed to the analog inputs of the AX08 analog-to-digital converter of the LAB-8 computer system. The bandpass of the amplifiers at output to the averaging computer was .15-500 Hz.

Computation of the EBR was done by using a Digital Equipment Corporation LAB-8 computer system with advanced averager program. For each of the two simultaneously recorded channels, computations were made over 500 data points with a time base of 850 msec. Each EBR resulted from 240 stimulus presentations. At the beginning and end of each session, a 10-microV 100 -msec-duration square wave derived from a Grass SDS stimulator was fed through the system and averaged for calibration purposes.

$$
\text { PROCEDURE }
$$

Each $\mathbf{S}$ was studied in five sessions.
During each session, EBRs were computed at each of the five intensity levels studied. Intensity was varied in $10-\mathrm{dB}$ steps over a $40-\mathrm{dB}$ range, with highest intensity level being $85 \mathrm{~dB}$ SPL re .0002 dyne $/ \mathrm{cm}^{2}$. A random order of stimulus presentation was used for each session. A different random order was used for each session, with $\mathrm{S}$ uninformed as to presentation order. Thus, each of the five intensity levels was repeated five times for a total of 25 trials.

The Ss were instructed to remain relaxed but alert and to minimize body movements. Because the Ss were run late in the evening, the need to remain alert was especially emphasized. For all trials, the Ss were seated in a dental chair within a dark electrically shielded booth. A bell was rung to signal the beginning or end of a trial to the $\mathrm{Ss}$. Conversely, the $\mathrm{S}$ signaled, by activating a light with a hand-held pushbutton, when he was ready to begin. Upon receiving this "ready" signal from the $\mathrm{S}$, the $\mathrm{E}$ began the trial only when the monitored EEG was judged to be artifact free. A minimum of 5 min between each trial, during which the $S$ was free to move, given the constraints of the electrodes and chair, was scheduled. During this 5-min interval, the averaged EBR was plotted on the $X \cdot Y$ recorder and punched out on paper tape.

The EBRs were computed simultaneously from the monopolar site $C_{3}-A_{2}$ and from the bipolar site $\mathrm{Cz}-\mathrm{Oz}$. These averages were collected in real-time and then preserved via $X-Y$ plots and in digital form on punched paper tape. After all five sessions had been completed, the punched paper tapes were summed together, for each
$S$ at each intensity level, using a program developed in this lab. Measurements of the responses were made from the summed punched tape digital values, which were typed out on the computer's Teletype. RESULTS

When all sessions had been completed on all three Ss, two compendiums were computed for each $S$. These compendiums consisted of summing together the data from one recording for all 25 trials and plotting the obtained digital values by hand. An example of two compendiums for S H.B. is shown in Fig. 1. These compendiums provide a detailed view of the major components of the waveform. The amplitude measures $B$ to $C$ and $C$ to $D$ were measured in this study. Inspecting Fig. 1, one notes that the shape and latency of the two waveforms is almost identical. The primary difference between $\mathrm{C}_{3}-\mathrm{A}_{2}$ and $\mathrm{Cz} \cdot \mathrm{Oz}$ sites is in the size of the response. The monopolar recording gave, on the average, an EBR approximately 2-3 microV larger than did the bipolar. X-Y plots of individual EBRs computed for $\mathrm{S}$ H.B. during one session are shown in Fig. 2. An increase in amplitude of measures B-C and $C-D$ as intensity of stimulus increases is evident for both monopolar and bipolar recordings. Also evident in this data is the greater overall amplitude size of the monopolar EBR. The amplitude data were pooled, and plots of mean data points and regression lines are presented in Fig. 3. The linear

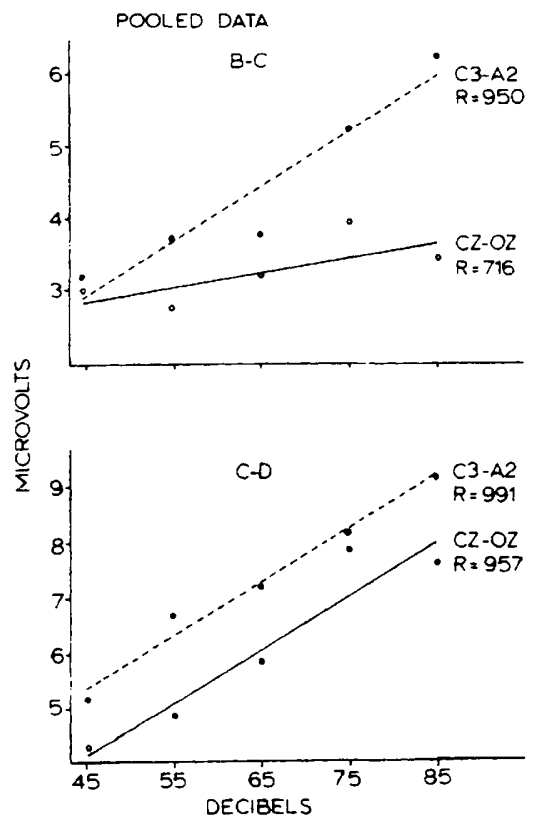

Fig. 3. Pooled amplitude measure for the three Ss. The circles represent mean data points and lines indicate linear regressions. 
regressions were fit to the pooled intensity functions by least-squares computation and correlation coefficients determined by the LAB- 8 computer system using the General Linear Regression program (DECUS No. 8-118). Examination of Fig. 3 reveals that the regression lines for Measure C-D had a steeper slope than did that for Measure B-C. In addition, the slopes for the C-D measure were identical for both recording conditions $(.094 \mathrm{microV} / \mathrm{dB})$. For the B-C measure, recording condition did appear to affect the slope of the regression line, with the monopolar recording giving a steeper slope (.076 microV $/ \mathrm{dB})$ than the bipolar $(.02 \mathrm{microV} / \mathrm{dB})$. The correlation coefficients followed a trend similar to the slope measures. Measure B-C, under the bipolar recording condition, shows the lowest correlation, and Measure C-D, under the monopolar recording condition, gives the highest correlation.

\section{DISCUSSION}

The results of this study suggest that the two recording conditions had only an indirect effect on auditory EBR intensity function measures. The amplitude measure used ( $\mathrm{B}$ to $\mathrm{C}$ or $\mathrm{C}$ to $D)$ to compute the intensity function appeared to play a major role in determining the degree of agreement between the two recording conditions. The simplest explanation of this effect may be that the amplitude $\mathrm{B}-\mathrm{C}$ measure from $\mathrm{Cz} \cdot \mathrm{Oz}$ is the smallest of the components measured. Therefore, the slope differences may be related to the lower signal-to-noise ratio. Some evidence for this conjecture is based on the results of the bipolar placement study previously reported (Tepas et al, 1971), which did not show any significant differences between Components B-C and C-D for the $\mathrm{Cz} \cdot \mathrm{Oz}$ recording. That study ran twice as many sessions as the present study, therefore, in effect, improving the signal-to-noise ratio. Thus, monopolar recordings may be desirable if large responses are desirable due to adverse signal-to-noise conditions or if repeated measures are not feasible.

Of particular interest in this study was the use of two techniques not generally used by other researchers in this area. One was summing of several repeated measures across sessions via punch paper tape and the measurement of these sums in a digital form. The second technique was the fitting of linear regression lines to the resulting intensity data. The slope of the regression line gives us a measure of how sensitive the EBR is to intensity changes, and the correlation coefficient shows how closely the relationship between changes in the EBR's amplitude and stimulus intensity fit a linear model. We also can note that the use of a wider auditory stimulus intensity range in the present study did not disturb the linear relationship previously reported by Tepas et al (1972) when they used a narrower range of intensities.

In summary, the results indicate that, for a clinical or other situation where the $E$ is unable to collect repeated measures and to average a large number of responses, a monopolar recording should be used. However, when the $\mathrm{E}$ can use repeated testing, precise stimulus control, and large EEG samples, either recording method is appropriate, bearing in mind that use of a lower amplitude measure, such as B-C, increases the need for more data collection. Until similar experiments are performed for the visual and somato-sensory systems, one should not assume that these results apply to other modalities. It would be interesting to use the technique of comparison of intensity function to test for differences between the myriad references that have been used by various Es doing monopolar recordings.

\section{REFERENCES}

GOFF, W. R., MATSUMIYA, Y. ALLISON, T., \& GOFF, G. D. Cross-modality comparisons of averaged evoked potentials. In $E$. Donchin and $D$. B. Lindsley (Eds.), Average evoked potentials. Washington, D.C: NASA, 1969.

JASPER, $H$ H. The ten-twenty electrode system of the International Federation. Electroencephalography \& Clinical Neurophysiology, 1958, 10, 371-375.

PERRY, N. W., \& CHILDERS, D. G. The human visual evoked response. Springfield, Ill: Thomas, 1969.

RECHTSCHAFFEN, A., \& KALES, A. (Ed.), A manual of standardized terminology, techniques and scoring system for sleep stages of human subjects. Washington, D.C: Public Health Service, U.S. Government Printing Office, 1968.

TEPAS, D. I., BOXERMAN, L. A., \& ANCH, A. M. Auditory evoked brain response: Intensity functions from bipolar human scalp recording. Perception \& Psychophysics, 1972, 11. 217-221.

VAUGHAN, H. G. The perceptual and physiologic significance of visual evoked responses recorded from the scalp in man. In $H$. Burian and J. Jacobson (Eds.), Clinical electroretinography, Proceedings 3rd International Symposium. New York: Pergamon Press, 1966. NOTES

1. The letters $B, C$ and $D$ have been used to identify the components measured in this study to avoid nomenclature difficulties that might arise in the reporting of other studies, which we plan to publish, dealing with paired stimuli. The components B, C, and $D$ are often referred to in the literature as $P_{1}, N_{1}$, and $P_{2}$, respectively.

2. The authors wish to acknowledge the cooperation of the Gentex Corporation, Carbondale, $\mathrm{Pa}$., in providing the earphones and headset assembly used in this study. 